\title{
BINARIES IN GLOBULAR CLUSTERS
}

\author{
S. L. W. MCMILLAN \\ Drexel University \\ Department of Physics, Philadelphia, PA 19104, U.S.A. \\ C. PRYOR \\ Rutgers, the State University of New Jersey \\ Dept. of Physics and Astronomy, 136 Frelinghuysen Rd., Piscataway, NJ 08854, U.S.A. \\ AND \\ E. S. PHINNEY \\ California Institute of Technology \\ Theoretical Astrophysics, 130-33 Caltech, Pasadena, CA 91125, U.S.A.
}

\section{Dynamics of Binary Stars (SLWM)}

Binary stars in a globular cluster (hereafter, GC) may be primordial (i.e. formed along with the cluster), or the result of cluster dynamics. "Dynamical" binaries can result from conservative threebody encounters (e.g. Spitzer, 1987) if a third star can carry away enough kinetic energy to leave two others bound, or from dissipative two-body encounters, if two stars happen to pass within a few stellar radii of one other (Fabian, Pringle, \& Rees, 1975). Such non-primordial systems are likely to be found primarily in evolved GC cores, both because conditions are more favorable for making them there, and because of mass segregation. Knowledge of the formation process allows reasonable estimates to be made of their mass and energy distributions. The initial spatial, mass, and energy distributions of primordial binaries, on the other hand, are largely unknown.

Until the late 1980s, the conventional wisdom was that GCs were born with few, if any, binary systems, so GC binaries had to form dynamically and consequently were rare. However, there is now strong evidence that many GCs contain binary fractions in the $\sim 3-30 \%$ range (see $\S 2$ ), and it seems reasonable to suppose that most, and quite possibly all, GCs began their lives containing significant numbers ( $\gtrsim 10 \%$, say) of primordial binaries.

BINARIES and GLOBULAR CLUSTER EVOLUTION. The role of binaries as energy sources driving GC evolution is well known (see, e.g. Heggie, 1975). Briefly, hard binaries (i.e., systems having binding energies exceeding the mean kinetic energy $\frac{3}{2} k T$ of cluster stars) tend to become more tightly bound following interactions with other cluster members. For equal-mass systems, the median fractional energy increase is $\delta E / E \sim 20 \%$; the mean time between interactions scales inversely with binary energy $E$, so the time-averaged heating rate stays constant, at about $1 k T$ per local relaxation time. The details depend somewhat on the masses of the stars involved-in particular, the energy release shows systematic trends with both the binary mass ratio and the mass of the incoming star-but the overall energetics are clear.

Numerical simulations have amply demonstrated how primordial binaries, if present at more than the $\sim 5 \%$ level, rapidly segregate to the cluster core and dominate both the core mass and the cluster evolution (McMillan, Hut, \& Makino, 1990, 1991; Gao et al., 1991; Heggie \& Aarseth, 1992). Even a small (1-2\%) initial population of hard binaries can give rise to a core binary fraction in the 10-20\% range within a half-mass relaxation time (a few billion years in typical cases). Subsequently, binaries control the cluster dynamics until they are all destroyed by interactions with other binaries, or recoil out of the cluster after a triple or four-body encounter.

The rate at which binaries interact and are depleted in the core is largely independent of the rate at which Galactic tides strip mass from the cluster's outer regions. As a result, there is a 
"watershed" initial binary fraction (of around 10-15\% for the simple models considered so far), below which the cluster binary fraction reaches zero before the cluster dissolves, and above which the binary fraction first falls, then rises prior to eventual dissolution (McMillan \& Hut, 1994). Even if their initial binary fraction is below this watershed value, however, the binary depletion time for many clusters may exceed the age of the universe.

Primordial binaries stabilize the core against collapse at a radius of a few percent of the halfmass radius. As a result, it seems quite unlikely that a GC will ever reach densities high enough for 3-body dynamical binaries to form, although 2-body tidal binary formation remains a possibility. Monte-Carlo simulations by Hut, McMillan \& Romani (1992) and Sigurdsson \& Phinney (1995) indicate that, at any instant, some non-negligible fraction of cluster binaries will be found well beyond the half-mass radius, drifting back toward the core after an interaction whose recoil did not quite eject them from the cluster's potential well.

It is well established that mass and angular momentum transfer in isolated binaries can lead to evolutionary pathways not accessible to single stars. When interactions with other stars enter the mix, still more possibilities arise. "Resonant" encounters with other stars or binaries almost invariably lead to very close approaches between some pair of stars, so the presence of binaries in a cluster can greatly increase the probability of stellar collisions and close encounters, particularly in low-density systems (Verbunt \& Hut, 1987). Even without physical collisions, binary interactions can profoundly affect binary evolution. "Flyby" encounters may radically change a binary's orbital parameters, while "exchange" interactions, by preferentially ejecting the lightest of the stars involved, can lead to systematic and largely irreversible changes in overall binary composition.

SIMULATIONS of BINARY-RICH STAR CLUSTERS. The presence of a substantial primordial binary population leads to an intimate coupling of cluster dynamics and stellar evolution. Binaries control the dynamics, but the cluster environment strongly influences each binary's evolution and survival probability. Over time, the most massive objects in a cluster tend to find their way into binaries in or near the core; the binaries then mediate interactions between these objects, possibly producing many of the exotica observed in GCs today (see §3). Perhaps surprisingly, given the important connection between observations and our understanding of cluster dynamics, theoretical simulations of cluster dynamics have tended to stop short of including stellar-evolutionary effects. To date, no fully (or even nearly) self-consistent calculation including both dynamics and evolution has ever been carried out.

One reason for this is the "kitchen sink" effect that follows when one contemplates moving beyond the simplest simulations. For the most part, model systems incorporating binaries have consisted of identical, non-evolving point masses - a good starting point, but hardly an accurate model of any real star cluster. Such a simple system has only one relevant parameter - the total number of stars, N. However, when we add a spectrum of stellar masses, we necessarily introduce real stellar physics into the calculation - a stellar mass function must be chosen, and the spatial distribution of each stellar species defined. Once this is done, it immediately becomes necessary also to include the effects of stellar evolution - stellar evolutionary time scales are often comparable to the relaxation/core collapse time of the cluster, so the dynamical state of the cluster is necessarily coupled to the state of the component stars. Stellar evolution drives binary evolution, so this too must be incorporated if the simulation is to remain self-consistent, and the seemingly innocuous act of including stellar masses actually leads inevitably to a complex mix of physical processes and a vastly more difficult numerical problem.

Over the past few years, a number of groups have been gearing up to meet this challenge. It seems clear that "pure" large-N techniques, such as Fokker-Planck and gas-sphere codes, are structurally incapable of following systems containing large numbers of evolving binaries and evolution products. However, when coupled with Monte-Carlo treatments of binaries (see Spurzem, these proceedings), these methods may regain applicability to binary-rich systems. Giersz (these proceedings) has recently reported significant progress in full Monte-Carlo cluster simulations, with and without binary populations. On the N-body side, Aarseth's venerable NBODY4 (Aarseth, 1996) has recently been joined by the "kira" integrator developed by McMillan, Hut, Makino, and Portegies Zwart as part of the Starlab software environment (see McMillan \& Hut, 1996). Both NBODY4 and kira include sophisticated treatments of stellar and binary evolution as integral parts of the programs (see Tout and Portegies Zwart, these proceedings; also Portegies Zwart et al, 1998). 


\section{Observations of Binary Stars (CP)}

This section briefly discusses some recent results constraining the number and properties of binary stars in GCs. More complete reviews of this field are Hut et al. (1992; H92 hereafter), Pryor et al. (1996), and Meylan \& Heggie (1997). Most of the information on the GC binary population comes from systematic surveys for eclipsing binaries, for radial velocity variables, and for stars above the main sequence in the color-magnitude diagram (CMD), also called "binary sequences." See H92 for an extensive discussion of the techniques used in these surveys. Exotic stellar objects in GCs, which are discussed in $\S 3$, are powerful probes of the interplay between binaries and GC dynamical evolution, but are less useful as guides to the total binary population.

Eclipsing binaries are rare in GCs, but present (see Mateo, 1995 and $§ 9.6$ of Meylan \& Heggie, 1997 for reviews). The frequency of these close binaries is probably an interesting probe of GC dynamics, but a poorer guide to the total binary population. Contact (W UMa) systems are the most easily detected and primordial binaries with periods shorter than perhaps five days are expected to have been brought into contact over the age of the GCs by the angular momentum loss associated with magnetized winds (Eggen \& Iben, 1989). Unfortunately, the frequency of W UMa systems is difficult to compare to that of the other binary populations because W UMa lifetimes are uncertain by about a factor of 100 (Eggen \& Iben, 1989). Indeed, it now probably more fruitful to use GC binary frequencies to constrain these lifetimes rather than vice versa.

Most surveys for radial velocity variables in GCs have studied luminous giants and only been sensitive to orbital periods $\left(P_{\mathrm{s}}\right)$ in the two-decade range between 0.2 and 20 years. The lower limit comes from the smallest orbit in which the giants can fit without Roche-lobe overflow and the upper limit from the time span of the observations and their accuracy. These studies find that an average of 0.07 of the stars in GCs are the primary of a binary with a period in an arbitrary decade within the above range and a mass ratio larger than 0.22 (see Pryor et al., 1996 and H92 for the details). Statistical and systematic uncertainties allow this fraction to be 0.03-0.15.

This GC binary frequency can be compared to the value of 0.06 per decade of period for $P \approx 2$ yr found for local solar-type stars (Duquennoy \& Mayor, 1991). It is surprising that GCs are not obviously deficient in binaries compared to the Population I field, given that binaries are expected to be destroyed by dynamical processes in GCs (Heggie, 1975, H92, §1). However, the period ranges that are most vulnerable to elimination are those with $P \lesssim 5$ days, as noted above, and those with

$$
P \gtrsim(65 \mathrm{yr})\left(5 \mathrm{~km} \mathrm{~s}^{-1} / \sigma\right)^{3},
$$

where $\sigma$ is the cluster velocity dispersion (e.g. Pryor et al., 1996). These long-period binaries have binding energies smaller than the typical kinetic energy of stars in the cluster and are expected to be quickly disrupted (Heggie, 1975). The cluster must also be sufficiently dense for all of these "soft" binaries to have suffered an encounter; see, for example, Pryor et al. (1996).

Recent radial-velocity surveys have increased the range of orbital periods that can be detected either by increasing the time baseline or precision of the measurements (Mayor et al., 1996 $\omega$ Cen; Côté et al., 1996 - M22) or by observing less luminous giants (Barden et al., 1995 - M71; Yan \& Cohen, 1996 - NGC 5053) or actual main-sequence stars (Côté \& Fischer, 1996 - M4). The searches for short-period binaries found binary frequencies comparable to or larger than the previous average value. The searches for long-period binaries in $\omega$ Cen and M22 found smaller values. This led Côté et al. (1996) to suggest that the expected destruction of long-period binaries had been detected. This seems likely, but larger surveys in individual GCs and a better knowledge of the orbital ellipticity distribution (an important systematic uncertainty, see H92) are needed to be certain.

A possible reason why the present-day frequency of hard binaries in GCs is not clearly below that of the Pop I field is provided by the increasing evidence for enhanced binary frequencies in GCs which have lost a lot of mass to the galactic tidal field. As discussed in $\S 1$, mass segregation and tidal mass loss can increase the binary frequency (McMillan \& Hut, 1994). The most spectacular confirmation of this prediction is the open cluster NGC 3680, for which a careful radial velocity and proper motion survey found no main-sequence members fainter than 1.5 mag below the turnoff (Nordström \& Andersen 1997). This suggests that 90\% or more of the initial cluster mass has been lost for reasonable initial mass functions. Over $50 \%$ of cluster members are found to be binaries, a much larger fraction than has been found by comparable surveys of Population I field stars. 
Among the GCs, M71 has a low total mass and an orbit confined to the galactic disk, suggesting that it has suffered extensive tidal mass loss, and has a binary fraction at the upper end of the observed range (see Pryor et al., 1989 and Côté et al., 1996). Veronesi et al. (1996) estimate that the very low luminosity cluster E3 has a binary frequency (including all orbital periods) of about $30 \%$ based on a binary sequence in the CMD. Clearly, it will be interesting to look for a correlation between binary frequency and indicators of the amount of tidal mass loss.

Searches for binary sequences benefit from the high angular resolution of the Hubble Space Telescope (HST), which reduces the number of coalesced stellar images, caused by crowding, that mimic binaries (see Romani \& Weinberg, 1991, H92). Rubenstein \& Bailyn (1997) studied the postcore-collapse (PCC) GC NGC 6752 and found a binary frequency of 15-38\% (99.7\% confidence interval) within $11^{\prime \prime}$ of the center and $0-16 \%$ beyond. These frequencies include binaries with all periods that are present. These numbers and the CMDs themselves suggest that the binaries are strongly concentrated towards the cluster center, as would be expected from mass segregation (see $\S 1$ ). What is surprising is that there is such a large population of binaries near the cluster center at all. The projected radius containing half of the cluster light is about 140" (Harris, 1996), so, as discussed in $\$ 1$, the binary population probably should have maintained a resolvable core. Detailed dynamical modeling of NGC 6752 would be interesting. Searches for binary sequences also need to carried out in the centers of other PCC GCs.

Fahlman et al. (1997) have estimated the binary frequency in the nearby non-PCC GC M4 using the CMD derived from HST data extending from the center out to about 6 core radii. The binary frequency is 0.05 with a statistical uncertainty of $\pm 30 \%(2 \sigma)$ and there is some evidence that the frequency increases inward. This value is no higher and probably lower than the average GC binary frequency from radial velocity surveys, despite being sensitive to a much wider range of periods. This perhaps suggests that a narrower range of binary periods has survived than predicted by theory. An extensive radial velocity survey is underway in M4. A prediction of the properties of the binary population expected to have survived in the cluster would also be useful.

\section{Exotic Stars and Binaries in Globular Clusters (ESP)}

The mass function of non-degenerate stars in GCs now extends only to $\sim 0.8 M_{\odot}$. However the dynamical and chemical evolution of clusters depends crucially on much more massive stars now present only as cold relics: black holes, neutron stars and white dwarfs. These are detectable only through their gravitational effects unless lit up by accretion from a binary companion. In a bright cluster of $L_{V} \sim 10^{6} L_{V \odot}$ (e.g. M15, 47 Tuc), extending a Salpeter initial mass function (IMF) gives 5000 neutron star progenitors $\left(12-25 M_{\odot}\right), 2000$ black hole progenitors $\left(25-60 M_{\odot}\right)$, and $\sim 3 \times 10^{5}$ white dwarfs. The whole GC system has about 20 times this luminosity, about $10^{-3}$ of the luminosity of the Milky Way. Yet the clusters contain $10^{-1}$ of the low-mass X-ray binaries in the Galaxy (the same ratio applies to the X-ray sources in M31 and its GCs, (Supper et al., 1997)), and about half of the known recycled pulsars. In contrast, there are no black hole candidates in clusters, and cataclysmic variables have been hard to find, and are clearly not so overabundant as $\mathrm{X}$-ray binaries and recycled pulsars.

These systematics, and the properties of the binaries (particularly the pulsars) are determined by binary interactions and their back reaction on cluster evolution. These exotic binaries still provide our main observational evidence for binary interactions and their importance in cluster evolution.

BLACK HOLES. There is no evidence for black holes in GCs. The radial distribution of pulsars in the center of M15 limits the number of $\sim 10 M_{\odot}$ black holes in that cluster to $<100$ (Phinney, 1993). Thermonuclear bursts from all ten of the well-studied X-ray sources in GCs rule out the possibility that they are accreting black holes. This is at first quite surprising. If stars more massive than $25 M_{\odot}$ create black holes, then for every neutron star, 0.4 black hole should be created in an $x=1.35$ Salpeter IMF ( 0.1 would be created for $x=3$ ). The retention fraction, dynamical friction and exchange cross-section should all be larger than for neutron stars. So why have no black hole $\mathrm{X}$-ray binaries been seen? A likely explanation is that the clusters were born with many black holes, but that these rapidly segregated to the cluster cores, formed binaries, and ejected themselves by binary interactions (Sigurdsson \& Hernquist, 1993; Kulkarni et al., 1993). A few could be left, particularly in lower density clusters, and might appear as X-ray transients. 
NEUTRON STARS and WHITE DWARFS. The properties of cluster X-ray binaries have been reviewed by Verbunt (1996) and Bailyn (1996) and those of cluster radio pulsars by Phinney (1996). At birth, neutron stars are kicked to a velocity of $\sim 300 \mathrm{~km} \mathrm{~s}^{-1}$ (Hansen \& Phinney, 1997), much higher than the escape velocity from GCs. How were cluster neutron stars retained for recycling? Perhaps from a low-velocity tail of the kick distribution, but binaries are probably the main source. If the more massive star in a close circular binary goes supernova (the progenitor of a low-mass $\mathrm{X}$-ray binary [LMXB]), the binary will remain bound only if the remnant neutron star is kicked with almost the same speed and direction as the low-mass companion. Thus the center of mass velocity will be about equal to the (high) pre-supernova relative orbital velocity, explaining why Galactic LMXBs and low-mass binary pulsars are rare and fast-moving. But if the less massive star goes supernova (typical of high-mass X-ray binaries, where the initially more massive star undergoes unstable mass transfer, becomes the less massive one, and explodes as a helium star), the binary will remain bound mainly if the kick is about equal and opposite to the exploding star's orbital velocity (double and opposite flips the orbit over). The final center of mass velocity will be approximately the (low) pre-supernova orbital velocity of the massive companion. The subset of such systems in which there is no second spiral-in and supernova are the ones most likely to dominate neutron star retention in clusters. Specific models have been computed (Brandt \& Podsiadlowski, 1995; Kalogera, 1996; Drukier, 1996).

Today, the retained neutron stars are the most massive stars in GCs, so they tend to sink to the dense cores and to be preferentially left in binaries in 3 and 4 body exchanges (Sigurdsson \& Phinney, 1995; Heggie et al., 1996). This is why X-ray binaries and recycled pulsars are so overabundant in clusters relative to the Galactic disk. Though white dwarfs are much more numerous, they are less massive, so less centrally concentrated, and less likely to be retained in binary exchanges. This is probably why cataclysmic variables $(\mathrm{CVs})$ in clusters are not nearly so overabundant in clusters, though their numbers and formation mechanisms (Davies, 1997) are still poorly understood. Massive white dwarfs, which in the Galaxy seem to be preferentially magnetic (Sion et al., 1992), ought to be overrepresented.

The next few years should show great growth in the numbers of exotic objects available for study. Since 1994 the number of CV candidates in GCs has risen from 2 to 13: NGC6397 [3] (Cool et al., 1995; Grindlay et al., 1995), 47 Tuc [4] (Paresce \& De Marchi, 1994; Edmonds et al., 1996; Minniti et al., 1997; Shara et al., 1997), M4 [1] (Kałuzny, 1997), M5 [1] (Hakala et al., 1997), NGC6624 [1] (Shara et al., 1996), NGC 6752 [2] (Bailyn et al., 1996), M80 [1] (Shara \& Drissen, 1995). With the refurbished Arecibo, GBT and GMRT coming on line, the number of radio pulsars should grow dramatically. The increased angular resolution of AXAF should help resolve questions about the nature of clusters' low luminosity X-ray sources.

STARS. Nondegenerate stars are not immune from exchanges and two and three-body tidal encounters. Stars that have been proposed as candidate victims include high velocity stars in 47 Tuc (Meylan et al., 1991) [exchange], bright blue stragglers (Bailyn \& Pinsonneault, 1995; D'Antona et al., 1995) [collisional mixing], rapidly rotating horizontal branch stars (Peterson et al., 1995; Cohen \& McCarthy, 1997) [mergers], extended blue horizontal branch stars (Sosin et al., 1997; Rich et al., 1997) [envelope heating or stripping], and the missing red giants in the cores of corecollapse clusters (Djorgovski et al., 1991; Burgarella \& Buat, 1996) [envelope stripping], though the numbers and radial distributions of objects cause difficulties for many of these explanations.

Acknowledgments: SLWM's research has been supported by NASA grant NAGW-2559 and NSF grant AST 93-08005. CP's research on GCs is supported by NSF grant AST 96-19510. ESP's research has been supported by NASA grant NAG 5-2756 and NSF grant AST 96-18537.

\section{References}

Aarseth, S. J. (1996) in The Origins, Evolution and Destinies of Binary Stars in Clusters, eds. E.F. Milone \& J.-C. Mermilliod (San Francisco: ASP), 423

Bailyn, C.D. (1996) in The Origins, Evolution and Destinies of Binary Stars in Clusters, eds. E.F. Milone \& J.-C. Mermilliod (San Francisco: ASP), 320

Bailyn, C.D. \& Pinsonneault, M.H. (1995) ApJ, 439, 705

Bailyn, C.D. et al. (1996) ApJ, 473, L31 
Barden, S.C., Armandroff, T.E. \& Pryor, C. (1995) in The Origins, Evolution and Destinies of Binary Stars in Clusters, eds. E.F. Milone \& J.-C. Mermilliod, (San Francisco: ASP), 89

Brandt, N. \& Podsiadlowski, P. (1995) MNRAS, 274, 461

Burgarella, D. \& Buat, V. (1996) A\&A, 313, 129

Cohen, J.G. \& McCarthy, J.K. (1997) AJ, 113, 1353

Cool, A.M., Grindlay, J.E., Cohn, H.N., Lugger, P.M. \& Slavin, S.D. (1995) ApJ, 439, 695

Côté, P. \& Fischer, P. (1996) AJ, 112, 565

Côté, P., Pryor, C., McClure, R.D., Fletcher, J.M. \& Fischer, P. (1996) AJ, 112, 574

D'Antona, F., Vietri, M. \& Pesce, E. (1995) MNRAS, 272, 730

Davies, M.B. (1997) MNRAS, 288, 117

Djorgovski, S., Piotto, G., Phinney, E.S. \& Chernoff, D.F. (1991) ApJ, 372, L41

Drukier, G.A. (1996) MNRAS, 280, 498

Duquennoy, A. \& Mayor, M. (1991) A\&A, 248, 485

Edmonds, P.D. et al. (1996) ApJ, 468, 241

Eggen, O.J. \& Iben, I. (1989) AJ, 97, 431

Fabian, A. C., Pringle, J. E., \& Rees, M. (1975) MNRAS, 192, 15p

Fahlman, G.G. et al. (1997) submitted to ApJ

Gao, B., Goodman, J., Cohn, H., \& Murphy, B. (1991) ApJ, 370, 567

Grindlay, J.E. et al. (1995) ApJ, 455, L47

Hakala, P.J., Charles, P.A., Johnston, H.M. \& Verbunt, F. (1997) MNRAS, 285, 693

Hansen, B.M.S. \& Phinney, E.S. (1997) MNRAS, in press astro-ph/9708071

Harris, W.E. (1996) AJ, 112, 1487

Heggie, D. C. (1975) MNRAS, 173, 729

Heggie, D. C., \& Aarseth, S. J. (1992) MNRAS, 257, 513

Heggie, D.C., Hut, P. \& McMillan, S.L.W. (1996) ApJ, 467, 359

Hut, P., McMillan, S., Goodman, J., Mateo, M., Phinney, E.S., Pryor, C., Richer, H.B., Verbunt, F., \& Weinberg, M. (1992) PASP, 104, 981 (H92)

Hut, P., McMillan, S. L. W., \& Romani, R. (1992) ApJ, 389, 527

Kalogera, V. (1996) ApJ 471, 352

Kałuzny, J., Thompson, I.B. \& Krzeminski, W. (1997) AJ, 113, 2219

Kulkarni, S.R. Hut, P. \& McMillan, S. L. W. (1993) Nature 364, 421

Mateo, M. (1995) in The Origins, Evolution and Destinies of Binary Stars in Clusters, eds. E. F. Milone \& J.-C. Mermilliod, (San Francisco: ASP), 346

Mayor, M. Duquennoy, A., Alimenti, A., Andersen, J. \& Nordström, B. (1996) in The Origins, Evolution and Destinies of Binary Stars in Clusters, eds. E. F. Milone \& J.-C. Mermilliod, (San Francisco: ASP), 190

McMillan, S. L. W. \& Hut, P. (1994) ApJ, 427, 793

McMillan, S. L. W., \& Hut, P. (1996) ApJ, 467, 347

McMillan, S. L. W., Hut, P., \& Makino, J. (1990) ApJ, 362, 522

McMillan, S. L. W., Hut, P., \& Makino, J. (1991) ApJ, 372, 111

Meylan, G., Dubath, P. \& Mayor, M. (1991) ApJ, 383, 587

Meylan, G. \& Heggie, D.C. (1997) A\&A Rev, 8, 1

Minniti, D., Meylan, G., Pryor, C., Phinney, E.S., Sams, B. \& Tinney, C.G. (1997) ApJ, 474, L27

Nordström, B. \& Andersen, J. (1997) poster JD15-029P presented at the $23^{\text {rd }}$ IAU General Assembly

Paresce, F. \& De Marchi, G. (1994) ApJ, 427, L33

Peterson, R.C., Rood, R.T. \& Crocker, D.A. (1995) ApJ, 453, 214

Phinney, E.S. (1993) in Dynamics of Globular Clusters, eds. S. Djorgovski \& G. Meylan (San Francisco: ASP), 141

Phinney, E.S. (1996) in The Origins, Evolution and Destinies of Binary Stars in Clusters, eds. E.F. Milone \& J.-C Mermilliod (San Francisco: ASP), 163

Portegies Zwart, S., Hut, P., Makino, J., \& McMillan, S. L. W. (1998) to appear in Astronomy \& Astrophysics

Pryor, C. et al. (1996) in Dynamical Evolution of Star Clusters, IAU Symp. 174, eds. P. Hut \& J. Makino (Dordrecht: Kluwer), 193

Pryor, C., McClure, R.D., Hesser, J.E., \& Fletcher, J.M. (1989) in Dynamics of Dense Stellar Systems, ed. D. Merritt, (Cambridge: Cambridge University Press), 175

Rich, R.M. et al. (1997) ApJ, 484, L25

Romani, R.W. \& Weinberg, M.D. (1991) ApJ, 372, 487

Rubenstein, E.P. \& Bailyn, C.D. (1997) ApJ, 474, 701

Shara, M.M \& Drissen, L. (1995) ApJ, 448, 203

Shara, M.M., Zurek, D.R. \& Rich, R.M. (1996) ApJ, 473, L35

Shara, M.M., Bergeron, L.E., Gilliland, R.L., Saha, A. \& Petro, L.(1997) ApJ, 471, 804

Sigurdsson, S. \& Hernquist, L. (1993) Nature, 364, 423

Sigurdsson, S. \& Phinney, E.S. (1995) ApJSS, 99, 609

Sion, E.M., Fritz, M.L., McMullin, J.P. \& Lallo, M.D. (1988) AJ, 96, 251

Sosin, C. et al. (1997) ApJ, 480, L35

Spitzer, L. (1987) Dynamical Evolution of Globular Clusters, Princeton University Press

Supper, R. et al. (1997) A\&A 317, 328

Verbunt, F., \& Hut, P. (1987) in IAU Symposium 125, The Origin and Evolution of Neutron Stars, eds. D. J. Helfand \& J. H. Huang (Dordrecht: Reidel), 187

Verbunt, F. (1996) in Dynamical evolution of star clusters, eds. P. Hut \& J. Makino (IAU Symp 174), 183

Veronesi, C., Zaggia, S., Piotto, G., Ferraro, F.R. \& Bellazzini, M. (1996), in Formation of the Galactic Halo ... Inside and Out, ASP Conf. Series Vol. 92, eds. H. Morrison \& A. Sarajedini (San Francisco: ASP), 301

Yan, L. \& Cohen, J.G. (1996) AJ, 112, 1489 